\title{
Philosophiques
}

\section{Heidegger et la métaphysique : vers un double dépassement}

\section{Laurent Giroux}

Volume 2, numéro 2, octobre 1975

URI : https://id.erudit.org/iderudit/203031ar

DOI : https://doi.org/10.7202/203031ar

Aller au sommaire du numéro

Éditeur(s)

Société de philosophie du Québec

ISSN

0316-2923 (imprimé)

1492-1391 (numérique)

Découvrir la revue

Citer cet article

Giroux, L. (1975). Heidegger et la métaphysique : vers un double dépassement. Philosophiques, 2(2), 207-228. https://doi.org/10.7202/203031ar d'utilisation que vous pouvez consulter en ligne.

https://apropos.erudit.org/fr/usagers/politique-dutilisation/ 


\title{
HEIDEGGER ET LA MÉTAPHYSIOUE : VERS UN DOUBLE DÉPASSEMENT
}

\author{
par Laurent Giroux
}

Une lecture attentive des textes de Heidegger qui concernent directement la métaphysique nous a permis de déceler dans son discours une double ambivalence, - ce qui ne veut pas nécessairement dire ambiguïté ou antinomie, - la première touchant la notion même de 'métaphysique' et la seconde le mode de son dépassement possible. En effet, le terme 'métaphysique' désigne à la fois, chez Heidegger, le rapport à l'être même qui constitue l'essence de l'homme ${ }^{1}$, et l'oubli de l'être qui caractérise l'ontologie traditionnelle. D'autre part, le dépassement (Uberwindung) de la métaphysique se présente tour à tour soit comme un retour au fondement ou une fondation, soit comme une destruction ou déconstruction, soit encore comme le destin historique en vertu duquel la métaphysique serait d'elle-même surmontée ou vaincue.

Cette double ambivalence, dont nous allons voir qu'elle s'emboîte dans la dualité de l'existence authentique et inauthentique de l'être humain, telle que définie dans Sein und Zeit, crée une certaine confusion qui rend la lecture de Heidegger souvent laborieuse et sa compréhension ardue. L'analyse suivante voudrait être une manière d'avertissement contre les simplifications trop hâtives.

$\mathrm{Vu}$ que notre essai se présente comme un examen attentif des textes eux-mêmes pour les éclairer les uns par les autres et

1. La manifestation la plus immédiate de ce rapport est la compréhension spontanée que nous avons de l'être lorsque quelqu'un dit, par exemple: $\mathrm{Ce}$ merisier est (ou n'est pas). 
tenter d'en dégager une vue unitaire par delà l'ambivalence apparente ou réelle, nous serons forcés de multiplier les citations au risque d'alourdir quelque peu notre exposé. Ce dont nous nous excusons auprès du lecteur ${ }^{2}$.

\section{I - AMBIVALENCE DE LA MÉTAPHYSIQUE}

Sein und Zeit (1926) : \#\# 1-4, \# 6.

L'auteur de Sein und Zeit s'était donné explicitement pour tâche de poser à nouveau, c'est-à-dire d'une façon renouvelée (erneut) «la question du sens de l'être » $(S Z, 1)$. S'agit-il d'une reprise de la métaphysique?

Le mot 'métaphysique' apparaît dès la seconde ligne de l'introduction à Sein und Zeit, lorsque Heidegger affirme que «la question ci-dessus mentionnée est aujourd'hui tombée dans l'oubli, bien que notre époque compte comme un progrès de

2. Toutes nos références et nos citations sont extraites des textes originaux suivants et traduites par nous-même en français. Nous en donnons ici l'ordre chronologique.

8 avril 1926

Pentecôte, 1929

24 juillet 1929

Semestre d'été 1935: Einfübrung in die Metapbysik (M), Max Niemeyer Verlag( Tübingen, 1966.

1940: «Der europaïsche Nihilismus»;

1941: «Die Metaphysik als die Geschichte des Seins ;

«Entwürfe zur Geschichte des Seins als Metaphysik»; «Die Erinnerung in die Metaphysik 》, in NIETZSCHE II (N), Verlag Günther Neske,

1942-43: 《Hegels Begriff der Erfahrung 》 in Holswege (H), V. Klostermann, Frankfurt a. M., 1950.

1936-46: «Überwindung der Metaphysik», in Vorträge und Aufsätze I (VA), Verlag Günther Neske, Pfullingen, 1954.

1949: Einleitung zu Was ist Metapbysik?

1955: «Zur Seinsfrage», in Wegmarken (WM), V. Klostermann, Frankfurt, a. M., 1967.

24 février 1957 : «Die onto-theo-logische Verfassung der Metaphysik \$, in Identität und Differenz (ID), Verlag Günther Neske, Pfullingen, 1957. 
donner de nouveau son assentiment à la 'métaphysique' » (SZ, 2 ; cf. aussi \# 6, 21).

Heidegger semblerait donc voir une contradiction de son époque dans le fait de prétendre adhérer à la métaphysique tout en ayant perdu de vue la question du sens de l'être. Par la suite, le mot 'métaphysique' ne revient plus dans aucun des paragraphes de Être et temps où Heidegger montre la nécessité d'une reprise de la question du sens de l'être, ou bien expose les conditions de cette reprise. Seul le terme 'ontologie' y est employé, l'ontologie n'étant cependant pas identifiée tout simplement avec la métaphysique.

Nous réservons le titre ontologie pour l'interrogation théorique explicite touchant le sens de l'étant... (SZ, 12).

L'ontologie a pour objet de son investigation les concepts de base qui déterminent au préalable les différents domaines propres aux sciences ontiques (mathématique, physique, biologie, histoire, théologie, etc.), et de dégager la constitution d'être (Seinsverfassung) correspondante de l'étant qu'elles étudient.

Elle demeure cependant elle-même naïve et opaque, si ses recherches concernant l'être de l'étant laissent hors de discussion le sens de l'être pris absolument (den Sinn von Sein überbaupt)... La question de l'être vise par conséquent la condition a priori de possibilité non seulement des sciences qui examinent l'étant en tant que tel ou tel, et en cela se meuvent toujours déjà dans une certaine compréhension de l'être, mais la condition de possibilité des ontologies elles-mêmes qui précèdent et fondent les sciences ontiques $(S Z, 11)$.

Heidegger reconnaît ici à l'ontologie une double tâche: 1) Il dit que les recherches de l'ontologie concernent l'être de l'étant, ce qui dans notre première citation était désigné comme le sens de l'étant. Le sens de l'étant, objet de l'ontologie, serait donc son être (i.e. son mode d'être ou sa constitution d'être). 2) Il considère comme la tâche première de toute ontologie d'éclaircir d'abord le sens de l'être, comme condition de possibilité de l'ontologie elle-même. Cela semble assez paradoxal. D'après ce que nous avons vu plus haut, cependant, sur l'oubli de la question de l'être (à savoir l'inconséquence, pour Heidegger, d'une époque qui a des prétentions à la métaphysique sans se 
soucier de l'être), cette tâche pourrait être celle de la métaphysique, ce qui nous laisserait croire, au premier abord, à un dépassement des ontologies vers la métaphysique plutôt qu’à un dépassement de la métaphysique elle-même.

Ce que Heidegger appelle par la suite ontologie fondamentale, - définissant par là sa propre entreprise, - n'est rien d'autre que l'analytique existentiale du Dasein humain, en tant que compréhension pré-ontologique de l'être, et qui a pour but de rendre possible "l'amorce d'une problématique ontologique suffisamment fondée » et d'ouvrir ainsi une voie pour l'interprétation du sens de l'être (SZ, 13-14).

Cette nouvelle position de la question du sens de l'être que propose Heidegger présuppose une «destruction menée avec le fil conducteur de la question de l'être, du dépôt transmis par l'ontologie antique, ramenée aux expériences originelles où furent conquises les premières et désormais prédominantes déterminations de l'être » (SZ, 22). Dans tout ce passage, où la destruction coïncide avec le retour aux sources, on ne parle pas, encore une fois, de 'métaphysique', mais d'une «attestation (Nacbweis) de l'origine des concepts ontologiques fondamentaux 》 (SZ, 22).

L'objet que poursuit Heidegger se dégage maintenant avec une précision suffisante de ce qui précède : quoi qu'il en soit pour le moment de la position adoptée face à la métaphysique, - et qui n'est pas encore très claire, - la tâche de poser à nouveau la question du sens de l'ètre place l'auteur devant la nécessité de concevoir une sorte de méta-ontologie qui nous permettrait d'évaluer les ontologies et les concepts ontologiques depuis leur origine et leur fondement.

Kant und das problem der metapbysik (1929).

Einleitung, \#\# 1-2.

Le début de l'introduction de l'ouvrage bien connu de Heidegger sur Kant reprend, en somme, la perspective de Sein und Zeit, tout en y ajoutant certains éléments qui la rendent un peu plus complexe.

L'examen suivant se donne pour tâche d'interpréter la Critique de la raison pure de Kant comme une fondation de la méta- 
physique et de nous mettre sous les yeux le problème de la métaphysique comme étant celui d'une ontologie fondamentale. Ontologie fondamentale signifie cette analytique ontologique de l'être humain fini, qui doit préparer le fondement d'une métaphysique qui 'appartient à la nature de l'homme'. L'ontologie fondamentale est la métaphysique de l'existence (Dasein) humaine nécessairement requise pour rendre la métaphysique possible (K, 13):

Résumons le contenu de cette citation :

1) Le problème de la métaphysique est ramené à celui d'une ontologie fondamentale

2) L'ontologie fondamentale est définie comme plus haut dans Sein und Zeit.

3) L'ontologie fondamentale est la 'métaphysique' de l'existence humaine.

4) L'ontologie fondamentale doit poser le fondement de la métaphysique, "rendre la métaphysique possible».

5) La métaphysique est une 'disposition naturelle de l'homme'.

On remarquera que l'ontologie fondamentale qui, dans Sein und Zeit, avait pour but d'ouvrir la voie à une interprétation du sens de l'être, doit ici "préparer le fondement d'une métaphysique qui appartient à la nature de l'homme». Il semble donc que nous ayons affaire à une seule et même démarche. En second lieu, l'ontologie fondamentale constitue elle-même une pré-métaphysique, une métaphysique de l'homme destinée à « rendre la métaphysique possible ». Enfin, vu que l'auteur accepte sans discussion l'idée de Kant que la métaphysique appartient à la nature de l'homme comme une disposition essentielle, on peut difficilement supposer qu'il veuille détruire la métaphysique, du moins entendue de cette façon, ce qui serait détruire la nature de l'homme. Heidegger écarte d'abord l'idée qu'il s'agirait soit de lui donner un fondement, soit de lui donner un fondement nouveau qui remplacerait l'ancien, comme dans le cas d'un édifice déjà terminé et bien en place.

La fondation, c'est plutôt le projet du plan de construction luimême, de sorte que celui-ci indique du même coup sur quoi 
et comment l'édifice doit être fondé. La fondation de la métaphysique comme projet du plan de construction est la délimitation architectonique et la caractérisation de la possibilité interne de la métaphysique, c'est-à-dire la détermination concrète de son essence $(K, 14)$.

La tâche définie ici se ramène à la question de savoir: comment la métapbysique est-elle possible?, question qui reprend sans aucun doute celle qui avait été posée dans Sein und Zeit: comment l'ontologie est-elle possible?, et dont la réponse dépend de la possibilité d'un éclaircissement du sens de l'être. En toute évidence, nous sommes placés devant un plan de re-construction et non de dé-construction, comme on aurait pu s'y attendre. Dans «Zur Seinsfrage»(MM, 251), Heidegger présentera encore une fois la même entreprise comme un projet de construction (ein Bauen), ainsi que nous le verrons ci-après.

Bien que l'essence de la métaphysique demeure encore obscure, il ne s'agit pas, pour le moment du moins, de la supprimer, mais de «mettre à jour 'l'originarite' de son origine», rappelons-nous le retour «aux expériences originelles 》 (SZ, 22 ; WiM, 245), - ce qui n'est possible qu'en la reprenant à partir de sa fondation même $(K, 14)$ afin d'en évaluer la possibilité interne; Heidegger dira ailleurs : «d'en recommencer le commencement d'une façon plus originaire $(M, 30) »$.

Pour saisir ensuite quelle 'métaphysique' Kant recevait toute faite de la tradition, Heidegger emprunte la définition latine de Baumgarten, qui se traduit: «La métaphysique est la science qui contient les premiers principes de la connaissance humaine $(K, 15)$. $)$ Cette définition rejoint, sous une forme devenue épistémologique, la 'philosophie première' d'Aristote dont l'objet demeure ambivalent : soit la connaissance de l'étant en tant que tel (Tò ó $\nu \hat{\grave{\eta}} \partial{ }^{2}:$ Mét., 1003 a 21), soit celle du genre d'étant le

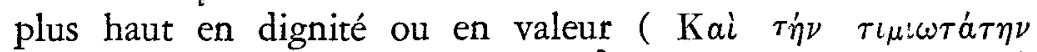

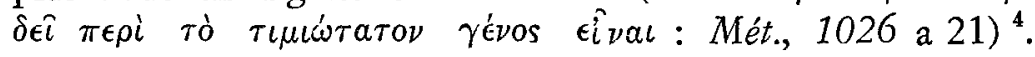

4. C'est sur la base de cette ambivalence que Heidegger désignera la métaphysique comme une onto-théo-logie (cf. ID, 49-52), où l'être, en tant que fondement de l'étant, s'estompe aussitôt et renvoie à l'Étant suprême comme à son propre fondement. "Dans l'unité de l'étant comme tel en général (Einheit des Allgemeinsten) et le plus élevé (Einbeit der Allbeit, d.b. des Höchsten) repose la constitution de l'essence de la métaphysique (ID, 49 et 52).» 
Ce rapprochement de deux définitions classiques permet ensuite à Heidegger de formuler à titre provisoire sa propre définition, qui n'est évidemment qu'un condensé des deux précédentes: «La métaphysique est la connaissance principielle (grundsätzliche) de l'étant comme tel et dans son ensemble ( $\mathrm{K}, 18$; cf. aussi ID, 49) » Ainsi comprise, la métaphysique n'est rien d'autre que l'ontologie traditionnelle. L'identité de l'ontologie et de la métaphysique, dans la mesure où celle-ci «dit ce que l'étant est en tant qu'étant» (= l'étantité ou vérité de l'étant), sera clairement exprimée dans la préface à Qu'est-ce que la métaphysique? (WiM, 19). De même dans Nietzsche II : «On peut donc circonscrire l'essence de la métaphysique, qui amène proprement l'étant à la parole et au concept, par le nom 'onto-logie' (N, II, 208). »

Mais l'auteur prend soin d'ajouter que sa définition est bien plus une indication du problème que pose la détermination de l'essence de la métaphysique que cette détermination même. Ce problème est ensuite ramené à trois questions principales, dont il est important de bien noter la formulation :

1) «En quoi consiste l'essence de la connaissance de l'être de l'étant?»

2) «Dans quelle mesure celle-ci se développe-t-elle nécessairement en une connaissance de l'étant dans son ensemble? »

3) «Pourquoi celle-ci culmine-t-elle à son tour en une connaissance de la connaissance de l'être ? 》

Le première question concerne la visée primordiale de la métaphysique, que Heidegger essaie de rejoindre; la seconde le développement historique de l'ontologie classique, et la troisième l'intention de la philosophie des temps modernes. Et Heidegger de conclure: «Ainsi la 'métaphysique' demeure-t-elle le titre pour l'embarras de la philosophie tout simplement 》 (für die Verlegenheit der Pbilosophie scblechtbin) (K, 18). Le problème de la métaphysique s'avère donc être, en fin de compte, le problème d'un problème, celui de la possibilité de cette science elle-même (K, 19). "Fondation de la métaphysique dans son ensemble signifie le dévoilement de la possibilité interne de 
l'ontologie $(K, 21)$ », c'est-à-dire, d'après les trois questions formulées ci-dessus, d'une connaissance de l'être (de l'étant) comme tel, voire d'une connaissance de cette connaissance (aspect critique). Ces trois questions suffiraient, à elles seules, à montrer que la préoccupation centrale de la pensée de Heidegger en est une d'ordre épistémologique : nous comprenons l'être. Comment cette compréhension est-elle possible? Est-il besoin de préciser que nous n'opposons pas 'épistémologique' à 'ontologique'. Le Versteben (le 'comprendre'), chez Heidegger, qui a son fondement dans l'Erschlossenheit (l'ouverture à l'être, litt. 'ouverturité'), est une dimension existentiale de l'être de l'homme. On a vite fait d'oublier que le connaître est un mode de l'être.

La façon dont Heidegerer croit que Kant a tenté de résoudre ce problème n'intéresse pas notre propos. Ce qui nous importe, c'est le rapport de l'ontologie à la métaphysique et le sens que Heidegger attache à leur dépassement possible.

Dans Sein und Zeit, l'ontologie a pour objet la constitution d'être des différents domaines de l'étant entre lesquels se partagent les sciences ontiques, tandis que la métaphysique, à peine mentionnée, semblerait viser le sens de l'être lui-même. Dans l'ouvrage sur Kant, où l'on se réfère explicitement à la division traditionnelle de la métaphysique en métaphysique spéciale, celle qui porte sur les grandes aires de l'étant (la nature, l'homme et Dieu), et en métaphysique générale ou ontologie, qui a pour objet l'étant en général ( $K, 18-19)$, Heidegger restreint sa définition provisoire à la métaphysique générale, c'est-à-dire à l'ontologie ou sens classique, qui étudie l'étant en tant que tel et dans son ensemble (étantité de l'étant - la seconde des trois questions formulées plus haut), alors que les deux autres questions qu'il pose mettent l'accent sur la connaissance même de l'être (de l'étant), introduisant par là un déplacement de la problématique. De quel ordre est cette connaissance? Dans son cours d" «Introduction à la métaphysique " (1935), Heidegger, sans donner de nom à sa propre entreprise, prétendra qu'elle est, de par son mode de questionnement, 'séparée par tout un monde' de l'ontologie classique $(M, 31)$. Ce en quoi elle se distingue sans doute des entreprises précédentes, c'est qu'elle va expliquer la connaissance de l'être par le passage au néant qui fonde l'existence humaine 
elle-même, comme nous le verrons tout de suite. Quant à l'originalité de cette démarche, il y aura lieu d'y apporter quelques nuances.

\section{Was ist matephysik (1929)}

La conférence intitulée «Qu'est-ce que la métaphysique?», qui date de $1929^{5}$, renonce dès le point de départ à « discourir sur la métaphysique ». Elle se place d'emblée «immédiatement dans la métaphysique», car "c'est seulement ainsi que nous lui procurons la véritable possibilité de se présenter elle-même» (WiM, 24).

Le fait que Heidegger pose de nouveau la question de l'essence de la métaphysique indique à la fois que cette question ne cesse de le préoccuper et qu'il demeure toujours incertain de la réponse. Que signifie alors se situer d'emblée à l'intérieur même de la métaphysique? La discussion que la conférence amorce porte sur le non-étant en tant que fondement de toute négation, qui se dévoile comme le 'rien' dans l'angoisse de l'existence et découvre du même coup l'étant en tant que tel : «qu'il est étant - et non rien 》 (WiM, 34). La post-face précisera en termes hégéliens ${ }^{6}:$ "L'absolument autre de tout étant est le non-étant (WiM, 45 ; cf. aussi «Zur Seinsfrage », WM, 246).» Ainsi l'étant et le néant vont-ils dialectiquement de pair, et cela, «parce que l'être même dans son essence est fini et ne se révèle que dans la transcendance du Dasein retenu au-delà dans le néant » (WiM, 40). Cet «être-par-delà-l'étant» (dieses Hinaussein ïber das Seiende) qui rend possible l'ouverture à l'étant comme tel, constitutive de l'existence humaine, Heidegger l'appelle la transcendance. Or, dit-il, cette transcendance, c'est la métaphysique même : "Ce passage par-delà l'étant, qui s'accomplit dans l'être du Dasein, c'est la métaphysique même (WiM, 41) ». Heidegger montre ailleurs ( $Z$ ur Seinsfrage », WM, 241) que le 'passage', appelé maintenant Überstieg (acte de frachir, trans-gression au sens étymologique), s'opère concrètement à l'intérieur d'une cer-

5. Nous faisons abstraction, pour le moment, de l'introduction et de la postface, qui sont tardives.

6. Ici, comme dans la section suivante, l'influence de la Logique de Hegel me paraît déterminante. 
taine représentation qui provient elle-même de l'étant et rebondit sur lui, sans préjudice, semble-t-il, de la pure transcendance du rapport à l'être qui rend possible toute métaphysique :

Partout la trans-gression qui revient sur l'étant est le 'transcendant absolument' (das transcendens scblecbthin), l' 'être de l'étant. La trans-gression est la métaphysique même, ce nom ne visant pas ici une discipline de la philosophie, mais cela qu' 'il y a' (es gibt) cette trans-gression... Que et comment 'il y a' l'être de l'étant, c'est la métapbysique au sens désigné (WM, 241 , souligné par nous).

Il demeure paradoxal que la transcendance vers le néant (= l'être), qui se trouve à l'origine de la connaissance de l'étant en tant qu'étant, c'est-à-dire la métaphysique, soit présentée dans les énoncés que nous avons soulignés comme la métapbysique même. Non seulement la transgression est la métaphysique, mais la trans-gression est l'être:

Une interrogation portant sur l'essence de la métaphysique ne peut avoir en vue que ce qui caractérise la métaphysique: cela, c'est la trans-gression: l'être de l'étant («Zur Seinsfrage», WM, 246).

Ainsi donc, il n'y a d'être que dans et par la trans-gression constitutive du Dasein comprenant l'être par-delà la connaissance immédiate de l'étant. C'est ce passage au-delà, cette trans-gression, qui produit l'étrangeté de l'étant d'où surgit tout 'pourquoi ?' et engendre l'étonnement exprimé dans la question métaphysique par excellence : «Pourquoi y a-t-il absolument de l'étant, et non pas plutôt rien? (WiM, 42). 》 Aussi lisons-nous dans Qu'est-ce que la métaphysique?: "L'homme seul parmi tous les étants éprouve, appelé par la voix de l'être, la merveille des merveilles: Que l'étant est (WiM, 46-47, 49).»

Einfïbrung in die metapbyrik (1935), I.

Ce que Heidegger reproche à la métaphysique traditionnelle, c'est d'avoir, en quelque sorte, posé ses assises sur cette 'merveille' sans jamais la thématiser pour elle-même, c'est-à-dire d'avoir succombé depuis l'origine à l'emprise de l'étant, laissant ainsi tomber dans l'oubli la question de l'être : 
(La métaphysique) part de l'étant et revient à lui. Elle ne part pas de l'être vers ce qui est digne de question dans sa 'manifesteté' (in das Fragwürdige seiner Offenbarkeit) (M, 65).

Ce qui vraiment étonne, ce qui suscite l'interrogation (ou devrait la susciter!) c'est que 'l'être' se manifeste. Autre formulation très voisine de la précédente dans la préface à Qu'est-ce que la métaphysique?:

Parce que la métaphysique interroge l'étant comme étant, elle s'arrête à l'étant et ne se tourne pas vers l'être comme être (WiM, 7).

On se rappellera que dans la conférence elle-même intitulée Qu'est-ce que la métaphysique?, la métaphysique était la transcendance vers l'être, tandis qu'ici, elle se caractérise par l'absence de cette transcendance, bien qu'elle "parle, dans ses réponses à la question de l'étant comme tel, depuis la 'manifesteté' inaperçue de l'être »; que "l'étant (lui) apparait chaque fois dans la lumière de l'être»(WiM, 8). Heidegger signale encore une fois la dominance voilée de l'être au cour même de l'oubli de l'être, de la Métaphysique au sein de la métaphysique, si bien que le terme 'métaphysique' demeure, de l'aveu même de l'auteur, «consciemment ambivalent». Mais cette ambivalence permet en même temps d'éclaircir la démarche de Heidegger. En effet, seul le questionnement portant sur le découvrement originaire de l'être, qui ouvrait la problématique de $\hat{E}$ tre et temps, peut jeter «une lumière sur l'essence jusqu'à présent cachée de la métaphysique 》 $(\mathrm{M}, 15)$. «'Introduction à la métaphysique' signifie par suite: introduire au guestionnement de la question fondamentale (M, 15) », celle que nous avons citée plus haut. Cette question exprime l'attitude interrogative qui «maintient l'étant au-delà dans la possibilité du non-être ».

L'étant n'est plus désormais ce qui est maintenant une fois làdevant (das nun einmal Vorbandene), il s'en trouve ébranlé... et y perd sa self-évidence comme étant (M, 21-22).

Il est remis en question de telle sorte que le questionnement lui-même prend une nouvelle forme :

Maintenant on est à la recherche d'un fondement qui doit fonder la domination de l'étant comme une conquête sur le néant 
(i.e. sur l'être) ... d'un fondement de la décision pour l'étant contre le néant $(M, 22)$.

La préface à Qu'est-ce que la métaphysique?, grâce à une expérience plus profonde encore de l'être dans le néant, reprend la question fondamentale en la serrant de beaucoup plus près :

D'où vient-il que partout l'étant a la préséance et réclame chaque 'est' pour lui, tandis que ce qui n'est pas un étant, le néant, ainsi compris comme l'être même, demeure dans l'oubli ? (WiM, 23; cf. aussi "Zur Seinsfrage », WM, 248).

Cette remise en question de la self-évidence de l'étant est à rapprocher de la critique que Hegel fait des objets de l'entendement en tant que choses en-soi, - objets 'abstraits' pour Hegel, - aussi bien dans la Différence des systèmes de Fichte et de Schelling que dans la Pbénoménologie et dans la Logique. L'entendement, dominé par la chose en soi ou l'étant, ne révèle pas l'être réel dans sa totalité. En outre, la sortie hors-de-soi du sujet, - révélée par la raison dialectique, — qui pose l'objet comme objet pour-soi, comme son être-autre, et le nie à la fois, est aussi une transcendance vers le néant ou l'être de l'objet. La dialectique est d'ailleurs définie comme «passage au-delà immanent (dies immanente Hinausgehen) par lequel l'unilatéralité et la limitation des déterminations de l'entendement se présentent pour ce qu'elles sont (i.e. dans leur être), à savoir leur (propre) négation »(Enzyklopädie, \#81).

Nietzsche II (1940-1941)

Cette primauté de l'étant marque pour Heidegger le début de la fin de la métaphysique.

Que signifie 'fin de la métaphysique'? Réponse: le moment historique en lequel les possibilités de l'essence (Wesensmöglichkeiten) de la métaphysique sont épuisées $(\mathrm{N}, 201)$.

L'achèvement de la métaphysique apparaît ici comme un moment de l'histoire et se trouve étroitement relié à l'essence de la métaphysique. Or, l'essence de la métaphysique en tant qu'ontologie (cf. N, 208) se caractérise, comme on l'a vu, par l'abandon de l'être, c'est-à-dire par la prédominance dominante de l'étant sur l'être, prédominance qui a son fondement dans la relation 
pré-ontologique de l'existant humain à l'étant en général, cette relation impliquant à son tour un rapport à l'être de l'étant. Le trait fondamental (l'essence) de la pensée métaphysique consiste à ramener ce double rapport à un seul, laissant dans l'ombre la relation à l'être même.

Est-ce que la raison de cette obscurité qui plane sur l'être et sur le rapport de l'homme à l'être ne serait pas la métaphysique et sa domination? Et quoi ? si c'était l'essence de la métaphysique d'ériger sans doute la vérité sur l'étant, et en cela de s'appuyer nécessairement sur le rapport de l'homme à l'être, mais sans réfléchir sur ce rapport lui-même, sans même pouvoir y réfléchir? $(\mathrm{N}, 207)$.

Comment alors Heidegger peut-il déclarer par ailleurs que sa démarche (appelée «der Schritt zurück»: le 'pas en arrière') «se meut bors de la métaphysique vers l'essence (ou la 'vérité') de la métaphysique »(ID, 41; VA, 71), et que «selon sa signification réelle, le terme métaphysique $\gg$ ne nomme rien d'autre que la connaissance de l'être de l'étant $\gg(\mathrm{N}, 220)$ ? Cette dernière citation ne fait pas vraiment difficulté, puisque, pour Heidegger, 'l'être' de l'étant et la 'vérité' de l'étant signifient la même chose et servent à désigner l'objet premier de la métaphysique occidentale. Il n'en demeure pas moins que l'essence de la métaphysique se dédouble ici et que c'est ce dédoublement qui rend possible la problématique ontologique (-épistémologique) de Heidegger, tout comme le dédoublement de l'existence humaine quant à sa compréhension de l'être (authentique et inauthentique) rend possible l'anthropologie de Etre et temps. L'idée d'une double essence de la métaphysique réapparaittra clairement dans «Zur Seinsfrage »:

Échapper à la métaphysique apparaît au premier abord comme un dépassement (Überwindung) qui ne fait que laisser derrière lui la représentation exclusivement métaphysique, pour conduire la pensée à l'air libre du triomphe sur l'essence de la métaphysique. Mais c'est seulement dans le triomphe que revient proprement de nouveau la vérité permanente de la métaphysique, apparemment repoussée, comme son essence désormais appropriée (WM, 244).

Ici, c'est sans doute sur la continuité de l'essence de la métaphysique que Heidegger met l'accent: la 'vérité permanente' de 
la métaphysique n'est qu' 'apparemment repoussée'. Mais cette continuité et cette permanence sous-tendent un mouvement noté comme un triomphe - entre l'essence non appropriée (inauthentique!) et 'l'essence appropriée' (authentique!) de la métaphysique. En fait, c'est toujours la même ambivalence dans laquelle l'auteur se maintient en cherchant son équilibre, ambivalence de la métaphysique saisie dans sa visée originelle, d'une part, et dans son blocage historique sur l'étant d'autre part. Cette tension interne entre l'être et l'étant constitue l'essence historique roncrète de la métaphysique ${ }^{7}$ :

L'être se distingue en être-tel (Was-sein: ce que la chose, l'étant, est) et en être tout court (Dass-sein: que la chose est, l'être). Avec cette distinction et sa préparation commence l'histoire de l'être comme métaphysique $(\mathrm{N}, 401)$.

Ainsi, ce que Heidegger appelle la 'différence ontologique', c'est-à-dire « la distinction de l'être et de l'étant, est-elle le fondement inconnu et non fondé, mais dont on se réclame néanmoins partout, de toute métaphysique $(\mathrm{N}, 210) \gg$ :

La différence de l'étant et de l'être est le domaine à l'intérieur duquel la métaphysique, la pensée occidentale dans la totalité de son essence, peut être ce qu'elle est (ID, 41).

Les derniers chapitres de l'ouvrage sur Nietzsche décrivent l'évolution du concept d'être en Occident, depuis son dévoilement originel dans l'alêtheia grecque jusqu'à sa fixation définitive comme actualité ou comme réalité, qui est sous-jacente à tous les modes de la pensée philosophique et scientifique d'aujourd'hui. C'est dans cette perspective seulement que peut se comprendre l'idée d'une «destruction de l'histoire de l'ontologie», i.e. des «recouvrements de l'essence initiale de l'être ${ }^{8}$, telle qu'annon-

7. Elle est également, selon Heidegger, l'essence même de la 'conscience naturelle' chez Hegel, qui correspond à la compréhension inauthentique de l'être dans Sein und Zeit, et finit par s'identifier avec la 'métaphysique absolue'. (CF. Holzwege, pp. 136-137.)

8. « La prédominance... de la détermination de l'être comme réalité est consolidée... La transmission de la vérité sur l'étant, qui suit son cours comme 'métaphysique' se développe sous la forme d'une accumulation, qui ne se reconnaît plus elle-même, de recouvrements de l'essence initiale de l'être. C'est en cela que la nécessité d'une 'destruction' de ce recouvrement trouve son fondement dès qu'une pensée de la vérité de l'être est devenue nécessaire ( $N, 415)$. 》 
cée dans Sein und Zeit. Encore une fois, cependant, la destruction du re-couvrement ne s'opère pas pour elle-même. Elle n'est que la négation inhérente à une dialectique du dé-couvrement, d'un retour au pur commencement ( $=$ origine, en un sens qui est temporal, mais pas nécessairement temporel) où l'être exerce son étance (sein Wesen treibt) :

L'histoire de l'être, connue historiquement comme métaphysique, a son essence en cela qu'une pro-gression (Fortgang) se produit hors du commencement. Dans cette pro-gression, l'être prend congé de lui-même dans l'étantité et récuse l'illumination de l'initialité (Anfängnis) du commencement... La pro-gression hors du commencement ne renonce pas à celui-ci, car, autrement, l'étantité ne serait pas un mode de l'être... La métaphysique est l'histoire de l'être comme pro-gression hors du commencement, cette progression faisant, une bonne fois, du retour un besoin et du rappel au commencement une nécessité urgente $(\mathrm{N}, 486-488)$.

Par rapport aux citations antérieures, les textes du Nietzsche insistent sur la dimension historique de la métaphysique ${ }^{9}$, sur la métaphysique en tant qu'histoire de l'être comme pro-gression hors de son origine, comme cheminement et acheminement qui annonce l'achèvement décrit dans "Überwindung der Metaphysik», dont le rapport avec le 'Scbritt zurück', le 'pas en arrière' (autre forme de l'Überwindung) aura à être défini dans notre seconde partie.

Dérive depuis son origine et exigence de retour à cette origine, telle est encore une fois l'essence historique concrète de la métaphysique, bien que son essence véritable consiste, selon

9. Rappel de quelques citations:

«La 'fin de la métaphysique' est le 'moment bistorique en lequel les possibilités de l'essence de la métaphysique sont épuisées' (N, 201).

Avec la distinction de l'être et de l'étant 'commence l'bistoire de l'être comme métaphysique' (N, 401).

«La transmission de la vérité sur l'étant qui suit son cours comme métaphysique... (N, 415) 》

«L'bistoire de l'être connue historiquement comme métaphysique... (N, 486-488) 》

«La métaphysique est l'bistoirt de l'être comme pro-gression hots du commencement... (486-488)》 
Heidegger, dans la transgression vers l'être qui sous-tend la progression dans l'étantité.

Une ambivalence parallèle gouverne l'idée de dépassement de la métapbysique.

\section{II - L'AMBIVALENCE DU DÉPASSEMENT}

Dans la postface tardive à Qu'est-ce que la métaphysique? Heidegger écrit :

La question 'qu'est-ce que la métaphysique?' interroge par-delà la métaphysique. Elle sourd d'une pensée qui s'est déjà engagée dans la voie de surmonter la métaphysique (WiM, 43).

D'autre part, la préface également tardive de 1949 porte le titre : Le retour au fondement de la métapbysique. Ce qui nous vient immédiatement à l'esprit, - suggéré du reste par le projet établi dans Sein und Zeit (\# 6, p. 22), - c'est que la tâche de surmonter ou de vaincre la métaphysique serait identique à celle d'en redécouvrir le fondement dernier, démarche qui, pour Heidegger, doit entraîner une transformation de la métaphysique elle-même. Vérifions cette hypothèse en rapprochant deux passages, l'un extrait de la postface, l'autre de l'introduction à Qu'est-ce que la métaphysique?

Première citation:

La métaphysique... dit ce que l'étant est, en ce qu'elle amène au concept l'étantité de l'étant. Dans l'étantité de l'étant, la métaphysique pense l'être sans pouvoir toutefois, selon son mode de penser, se pencher sur la vérité de l'être. La métaphysique se meut partout dans le domaine de la vérité de l'être, qui demeure pour elie le fondement non fondé et inconnu (cf. également p. 7 de l'introduction)... Alors s'impose la question de savoir ce qu'est la métaphysique dans son fondement. $\mathrm{Ce}$ questionnement doit penser métaphysiquement et, à la fois, depuis le fondement de la métaphysique, i.e. non plus métaphysiquement. Un tel questionnement demeure, en un sens essentiel, ambivalent (WiM, 44).

Seconde citation :

La métaphysique quitte constamment (son fondement). Mais elle ne lui échappe pourtant jamais. Dans la mesure où une 
pensée s'engage sur la voie d'expérimenter le fondement de la métaphysique, dans la mesure où cette pensée tente de penser à la vérité de l'être même au lieu de représenter seulement l'étant en tant qu'étant, la pensée a d'une certaine manière quitté la métaphysique. Cette pensée retourne, et cela toujours du point de vue de la métaphysique, au fondement de la métaphysique. Mais ce qui ainsi apparaît encore comme fondement est, sans doute, s'il est expérimenté à partir de lui-même, un autre encore non dit, si bien que l'essence de la métaphysique est autre chose que la métaphysique. (WiM, 8-9; c'est nous qui soulignons).

Dans «Zur Seinsfrage», Heidegger maintenait la "vérité permanente' de la métaphysique dans le mouvement même de l'appropriation de son essence. De même ici : aller au fondement de la métaphysique, c'est à la fois sortir de la métaphysique et y rester, c'est sortir sans en sortir, dé-couvrir 'l'autre' à la limite du 'même' comme sa négation, si l'on veut que le fondement fonde, fût-ce de façon négative, cela même qu'il fonde. L'ambivalence se ramène alors à un rapport dialectique, de type hégélien, entre la métaphysique et son origine, l'une niant l'autre pour se poser elle-même.

Une pensée qui pense à la vérité de l'être ne se contente certes plus de la métaphysique; mais elle ne pense pas non plus contre la métaphysique... La métaphysique demeure ce qui est premier dans la philosophie. Ce qui est premier dans la pensée, elle ne l'atteint jamais. Dans la pensée de la vérité de l'être, la métaphysique est surmontée... 'Surmonter la métaphysique' n'est pas éliminer la métaphysique. Aussi longtemps que l'homme reste l'animal rationale, il est l'animal metapbysicum. Aussi longtemps que l'homme se comprend comme le vivant raisonnable, la métaphysique appartient, selon le mot de Kant, à la nature de l'homme. Par contre, la pensée pourrait bien, si elle réussissait à retourner au fondement de la métaphysique, entraîner un changement dans l'essence de l'homme, changement qu'accompagnerait une transformation de la métaphysique (WiM, 8-9).

Compte tenu de ce que nous avons vu auparavant, surmonter la métaphysique serait donc saisir 'dans la pensée de la vérité de l'être' que l'élément premier de la philosophie n'est pas le fondement dernier de la pensée, le premier (la vérité de l'étant) 
demeurant enfermé dans l'ordre de la réalité objectale (des objets), le second (la vérité de l'être) rendant possible ('fondant') à la fois tout l'ordre objectal, c'est-à-dire l'étant dans son ensemble et la compréhension de l'étant en tant que tel.

En outre, le destin de l'homme, être métaphysique, est solidaire du destin de l'être (de sa vérité), solidaire, par conséquent, du destin de la métaphysique qui est le lieu historique du voilement de l'être. Heidegger prévoit qu'un retour au fondement de la métaphysique pourrait modifier l'essence même de l'homme et, par suite, donner naissance à une nouvelle métaphysique. Nous reviendrons à la fin sur des conditions de cette transformation.

Dégageons provisoirement le résultat des analyses précédentes. La pensée de la vérité de l'être rend compte à la fois de l'origine de la métaphysique et de son 'fatal' déracinement par rapport à son propre fondement, inconnu et méconnu, - cette origine et ce déracinement constituant son essence même, tout comme l'existence authentique du Dasein, en tant qu'ouverture à l'être, est l'horizon à partir duquel seule la quotidienneté inauthentique, tombée au pouvoir de l'étant, est rendue à la fois possible et intelligible. De même que l'existence inauthentique est incapable de penser son propre fondement depuis l'existentialité de l'être authentique, ainsi «la métaphysique comme telle (i.e. comme ontologie) est-elle, en vertu de sa propre essence, exclue de l'expérience de l'être » (WiM, 20), en lequel pourtant elle trouve son point d'appui. L'expérience de l'être et la connaissance de l'étant en général sont simplement d'un tout autre ordre, bien que la connaissance de l'étant comme tel ait son origine et son fondement dans l'expérience de l'être. Aussi «la pensée de la vérité de l'être, en tant que retour au fondement de la métaphysique, a-t-elle déjà, dès le premier pas, quitté le domaine de toute ontologie» (WiM, 21). Pourtant, aussi bien l'ontologie que l'expérience de l'être sont appelées tour à tour, par Heidegger, métaphysique.

\section{Überwindung der Metapbysik (1936-1946)}

Les réflexions groupées sous le titre «Überwindung der Metaphysik» poursuivent en l'explicitant l'idée déjà lancée dans 
Neitzsche II : l'Überwindung, c'est d'abord et avant tout le destin historique selon lequel la métaphysique se trouverait d'elle-même surmontée ou vaincue.

Elle est «l'événement appropriant (Er-eignis) par où l'être même est surmonté... La 'métaphysique' est déjà pensée comme destin de la vérité de l'étant, i.e. de l'étantité comme une appropriation (Ereignung) encore cachée, mais singulière, à savoir l'oubli de l'être... La métaphysique ne fait que commencer maintenant sa domination inconditionnée dans l'étant même et comme tel, sous la forme dépourvue de vérité du réel et des objets. Expérimentée depuis la lueur du commencement, cependant, la métaphysique est en même temps passée en ce sens qu'elle est entrée dans son achèvement (Ver-endung). L'achèvement dure plus longtemps que l'histoire antérieure de la métaphysique (VA, 63)».

En se constituant comme métaphysique par sa victoire incessante sur l'être, la métaphysique - telle est, vue sous un autre angle, l'ambivalence même de son essence - accomplit historiquement sa propre fin en succombant depuis l'origine à la domination inconditionnée de l'étant, comme réel et comme objet (sans vérité), et l'Überwindung n'est rien d'autre que cette longue agonie qui accompagne l'oubli de l'être. ${ }^{10}$ Elle se produit comme événement historique de l'appropriation de l'être sous l'empire de l'étant. Mais la «chute de la vérité de l'étant» va à son tour consacrer inéluctablement l'achèvement de la métaphysique:

La chute s'accomplit avant tout avec l'effondrement du monde marqué par la métaphysique et avec la dévastation de la terre qui provient de la métaphysique (VA, 64).

Par rapport aux autres écrits de Heidegger, la direction de l'Überwindung se trouve ici renversée. Il ne s'agit plus d'un 'pas en arrière', d'un retour au fondement de la métaphysique pour, en quelque sorte, la recommencer ou la refaire, mais de l'irréversible dérive de la métaphysique elle-même vers son ultime achèvement dans un monde rempli d'objets, mais privé d'être. Cette double direction de l'Überwindung n'est cependant pas contradictoire. C'est justement le sens que prend d'une façon de plus en plus évidente le destin de la métaphysique, - Nietzsche 
l'avait déjà bien aperçu, - qui contraint la pensée humaine à opérer d'urgence un retour aux sources et à envisager un nouveau commencement :

L'Überwindang est pensée ici en termes d'histoire de l'être. Elle est le signe du revirement inchoatif de l'oubli de l'être... Elle est la 'transmission' de la métaphysique vers sa vérité (VA, 70-71).

La métaphysique achevée, qui est le fondement du mode de penser planétaire, fournit la charpente pour un ordre de la terre qui doit sans doute durer longtemps. L'ordre n'a plus besoin de philosophie, car elle lui sert déjà de fondement. Mais avec la fin de la philosophie, la pensée n'est pas déjà aussi à son terme, mais en transit vers un autre commencement (VA, 75).

\section{Zur Seinsfrage (1955)}

Pour que ce transit soit possible, i.e. pour que la métaphysique accède à sa vérité, il semble toutefois qu'il faille quitter le langage de la métaphysique. Surmonter la métaphysique, c'est, avant tout, inventer un nouveau langage humain :

Mais la question de l'essence de l'être se meurt si elle n'abandonne pas le langage de la métaphysique, car la représentation métaphysique empêche de penser la question de l'essence de l'être (WM, 233).

Tout ici tient inévitablement au juste 'dire' à ce logos dont la logique et la dialectique, dérivées de la métaphysique, ne peuvent jamais expérimenter l'essence (WM, 237).

C'est sans doute en ce sens que Hegel, malgré tous ses assauts contre la choséité, a accompli la métaphysique, l'a poussée jusqu'à ses extrêmes conséquences sans en faire éclater le langage. Cependant, Heidegger affirme encore avec insistance que vaincre la métaphysique et son langage n'équivaut en aucune manière à une démolition de la métaphysique:

Il n'y a plus moyen de dépasser le fait grotesque que l'on proclame mes efforts de pensée comme une démolition de la métaphysique... Mais l'absence de réflexion a déjà commencé avec la mésinterprétation superficielle de la 'destruction' discutée dans 'Sein und Zeit' (1927), qui ne connaît aucune autre préoccupation que, dans la dé-construction ( $A b b a u)$ de représenta- 
tions devenues courantes et vides, de reconquérir les expériences originelles de l'être propres à la métaphysique (WM, 244-245).

La 'dé-construction' (Abbau) dont il est question ici n'est d'ailleurs que la phase négative d'une vaste entreprise de construction (Bau) que Heidegger décrit dans le même essai, et dont il n'est pas assuré de voir jamais le terme:

La métaphysique, de par son essence, ne permet jamais à la demeure humaine de s'établir proprement dans la localité (Ortschaft), i.e. dans l'essence de l'oubli de l'être. Aussi, la pensée et la poésie doivent-elles faire un retour là où elles ont, d'une certaine manière, toujours déjà été et, néanmoins, jamais encore bâti. Ce n'est toutefois, que par un bâtir (durch ein Bauen) que nous pouvons préparer la demeure dans cette localité. C'est à peine si un bâtir peut mijoter déjà sur l'érection de la maison pour le Dieu et d'un lieu de séjour pour les mortels. Il doit se contenter de bâtir sur la route qui ramène au lieu du dépassement de la métaphysique... (WM, 251).

Quant à la figure de ce lieu, de cette localité retrouvée, de la nouvelle demeure de la pensée par-delà la métaphysique et son langage, de cette humanité renouvelée dans son essence, 'désétantisée', 'déchoséifiée', 'dés-objectalisée', il faudrait interroger l'auteur lui-même; mais lorsqu'on admet n'être qu'en voie de bâtir, qu'en route vers le commencement, il est difficile de dire ce que sera l'édifice achevé et dans quel horizon de vérité sera définie sa structure architectonique.

\section{CONCLUSION}

Quelle conclusion tirer de toute cette analyse? Nous avons simplement voulu montrer que la pensée de Heidegger se meut sur deux plans, à la fois parallèles, complémentaires et interdépendants: d'une part, le plan fondamental de la transcendance vers l'être qui distingue l'existant humain en tant que tel et est considérée par Heidegger comme la métaphysique même; d'autre part, le niveau du rapport historique à l'étant où évolue l'ontologie traditionnelle sous ses diverses formes, sans pouvoir toutefois le ressaisir à sa source ('s'établir proprement dans la localité') dans la 'manifesteté' originaire de l'être, dont la présence-latente habite pourtant sans cesse les coulisses de l'oubli. 
Ce double niveau dialectiquement relié du rapport à l'être, qui crée l'ambivalence de la métaphysique chez Heidegger, commande également un dédoublement, sinon un 'triplement', dans le mode de dépassement possible de l'ontologie, conçu soit comme une redécouverte de la pure apérité de l'être par-delà l'étant ('préparer la demeure dans cette localité'), qui est l'essence même de la métaphysique et son fondement, soit comme une destruction ou 'dé-construction' des recouvrements successifs de ce 'donné' initial qui constituent l'essence concrète de la métaphysique, soit enfin comme le destin qui entraîne la métaphysique sur la pente raide de son auto-achèvement dans l'histoire, la prise de conscience de ce destin, - déjà amorcée, quoique d'une façon fort différente, par Hegel et Nietzsche, - se trouvant en fait à l'origine des deux premiers modes du dépassement.

La première étape à venir du dépassement définitif semble bien être celle de l'invention d'un nouveau langage - poétique peut-être - où le dieu pourrait habiter comme dans sa demeure et les mortels y séjourner.

Université de Sherbrooke

10. «La métaphysique est un sort au sens strict, - le seul entendu ici, - que, comme trait fondamental de l'histoire européenne occidentale, elle laisse l'humanité suspendue au milieu de l'étant, sans que jamais l'être de l'étant, comme la duplication (Zwiefalt) de l'un et de l'autre ne puisse être expérimenté et interrogé... dans sa vérité depuis la métaphysique et par elle (VA, 70)». 